\section{PET Prediction of Response to Trastuzumab in ErbB2-Positive Human Xenograft Model}

TO THE EDITOR: Recently, Kramer-Marek et al. (1) published a very interesting study about the use of Affibody-based PET to monitor response to trastuzumab in a human xenograft breast carcinoma model overexpressing ErbB2. The study provides specific information on ErbB2 receptor expression upon treatment.

We completely agree with the authors that noninvasive detection of ErbB2 expression by novel imaging molecules such as ErbB2specific Affibody molecules represents an important tool for in vivo identification of ErbB2-positive tumors and for significant information on disease dissemination, including brain metastasis. Moreover, in patients with ErbB2-negative primary breast tumors, this approach may enable identification of those with ErbB2-positive metastasis and thus relevant information in considering trastuzumab treatment.

Nevertheless, the authors' claim that the $N-\left[2-\left(4-{ }^{18}\right.\right.$ F-fluorobenzamido)ethyl]maleimide ( $\left.{ }^{18} \mathrm{~F}-\mathrm{FBEM}-\mathrm{HER}_{2: 342}\right)$ Affibody (Affibody $\mathrm{AB}$ ) may be used to determine whether trastuzumab treatment induces early ErbB2 downmodulation and a consequent decrease in efficacy of continued therapy should be carefully considered. The significant reduction of tracer uptake in human breast carcinoma BT474 xenografts after trastuzumab treatment could be related more to tumor size reduction than to ErbB2 downregulation. Indeed, although the authors observed a decrease in ErbB2 levels on the tumor cell membrane in some trastuzumab-treated mice, a large overlap of ErbB2 staining was also found between the trastuzumabtreated and control groups, suggesting that trastuzumab-induced ErbB2 internalization in the tumor cell is followed rapidly by receptor reexpression, with no global changes in ErbB2 levels. Consistent with this suggestion, a dramatic decrease of ${ }^{111}$ Indiethylenetriaminepentaacetic acid-trastuzumab and ${ }^{125} \mathrm{I}-\mathrm{C} 6.5$ diabody in trastuzumab-treated MDA MB-361 (2) and SKOV3 (3) xenografts, respectively, without ErbB2 downmodulation has been reported. Moreover, most immunohistochemical studies performed on ErbB2-positive tumors have revealed no changes in ErbB2 status after exposure to trastuzumab. In our pilot study of preoperative trastuzumab as monotherapy in patients with primary operable ErbB2-overexpressing breast tumors (4), the weekly administration of trastuzumab for 4 wk before surgery led to binding of ErbB2 receptors present on tumor cells but not to a change in ErbB2 expression levels; in all patients with residual tumor, the intense staining with biotinylated trastuzumab in the core biopsies was almost completely abrogated in the surgical specimens, whereas no change in ErbB2-positive staining was observed using monoclonal antibody CB11 directed against a synthetic peptide corresponding to the internal domain of ErbB2. In addition, no changes in ErbB2 receptor status were detected immunohistochemically in most patients with operable (5) or locally advanced (6) ErbB2-positive breast cancers after neoadjuvant exposure to trastuzumab in combination with

COPYRIGHT (C) 2012 by the Society of Nuclear Medicine and Molecular Imaging, Inc. chemotherapy. ErbB2-overexpressing tumors described as becoming negative on trastuzumab treatment appear to reflect the selection of ErbB2-negative cells rather than trastuzumabinduced ErbB2 downmodulation, as suggested by the loss of ErbB2 gene amplification detected by fluorescent in situ hybridization in $30 \%-40 \%$ of patients for whom enough residual tissue was available at the time of surgery to reassess ErbB2 after neoadjuvant therapy containing trastuzumab $(7,8)$. If xenografts in which the authors found significantly downmodulated ErbB2 expression were only those with loss of HER2 amplification and Affibody uptake, then Affibody-based PET may actually provide a useful strategy for monitoring tumor response to trastuzumab.

However, it should also be noted that trastuzumab-induced inhibition of tumor vascularization has been documented in preclinical models (9), and the authors described a correlation between ${ }^{18}$ F-FBEM-HER $2: 342$ Affibody uptake and high vessel count. Thus, impaired Affibody localization in xenografts may be due to decreased vascularization rather than to loss of ErbB2 expression.

Finally, tumor shrinkage induced by trastuzumab-containing therapy can sometimes be followed by an inflammatory reaction that masks any decrease in neoplastic mass. In those patients, a decrease in labeled Affibody uptake could be related to a reduced number of tumor cells instead of reduced ErbB2 levels, a hypothesis strongly supported by our recent report (10) of significant survival benefit in ErbB2-positive metastatic patients apparently nonresponsive on first-line trastuzumab treatment but in whom trastuzumab treatment was continued.

Overall, and in agreement with the authors, further intensive investigations are needed before one can conclude that this new noninvasive PET-based method represents a valuable strategy to evaluate changes in ErbB2 expression levels as an avenue to predicting response to trastuzumab.

\section{REFERENCES}

1. Kramer-Marek G, Gijsen M, Kiesewetter DO, et al. Potential of PET to predict the response to trastuzumab treatment in an ErbB2-positive human xenograft tumor model. J Nucl Med. 2012;53:629-637.

2. McLarty K, Cornelissen B, Scollard DA, Done SJ, Chun K, Reilly RM. Associations between the uptake of ${ }^{111}$ In-DTPA-trastuzumab, HER2 density and response to trastuzumab (Herceptin) in athymic mice bearing subcutaneous human tumour xenografts. Eur J Nucl Med Mol Imaging. 2009;36:81-93.

3. Reddy S, Shaller CC, Doss M, et al. Evaluation of the anti-HER2 C6.5 diabody as a PET radiotracer to monitor HER2 status and predict response to trastuzumab treatment. Clin Cancer Res. 2011;17:1509-1520.

4. Gennari R, Ménard S, Fagnoni F, et al. Pilot study of the mechanism of action of preoperative trastuzumab in patients with primary operable breast tumors overexpressing HER2. Clin Cancer Res. 2004;10:5650-5655.

5. Harris LN, You F, Schnitt SJ, et al. Predictors of resistance to preoperative trastuzumab and vinorelbine for HER2-positive early breast cancer. Clin Cancer Res. 2007;13:1198-1207.

6. Mohsin SK, Weiss HL, Gutierrez MC, et al. Neoadjuvant trastuzumab induces apoptosis in primary breast cancers. J Clin Oncol. 2005;23:2460-2468.

7. Mittendorf EA, Wu Y, Scaltriti M, et al. Loss of HER2 amplification following trastuzumab-based neoadjuvant systemic therapy and survival outcomes. Clin Cancer Res. 2009;15:7381-7388.

8. Hurley J, Doliny P, Reis I, et al. Docetaxel, cisplatin, and trastuzumab as primary systemic therapy for human epidermal growth factor receptor 2-positive locally advanced breast cancer. J Clin Oncol. 2006;24:1831-1838.

9. Spector NL, Blackwell KL. Understanding the mechanisms behind trastuzumab therapy for human epidermal growth factor receptor 2-positive breast cancer. J Clin Oncol. 2009;27:5838-5847. 
10. Campiglio M, Bufalino R, Sandri M, et al. Increased overall survival independent of RECIST response in metastatic breast cancer patients continuing trastuzumab treatment: evidence from a retrospective study. Breast Cancer Res Treat. 2011;128:147-154.

\section{Elda Tagliabue* \\ Manuela Campiglio \\ Andrea Balsari \\ Sylvie Ménard \\ *Fondazione IRCCS Istituto Nazionale dei Tumori \\ Via Amadeo 42 \\ 20133 Milan, Italy \\ E-mail: elda.tagliabue@istitutotumori.mi.it}

Published online Aug. 23, 2012.

DOI: 10.2967/jnumed.112.108068

REPLY: The work that we have recently described tested the feasibility of the use of Affibody molecules (Affibody $\mathrm{AB}$ ) and PET to predict tumor response to ErbB2-targeted therapy. It is clear that additional studies will be needed to dissect the mechanistic events underlying the observed changes. Differences among tumor cell lines could affect responses as well.

Clinical studies show that the assessment of ErbB2 level by immunohistochemistry produces variable results among laboratories. This variation may be due to differences in immunohistochemistry staining techniques and scoring criteria (1). For antigen-retrieval processes, the solution used (e.g., citrate buffer or ethylenediaminetetraacetic acid and their $\mathrm{pH}$ ), the duration of heating, and antigen retrieval may all affect detection of the ErbB2 antigen by immunohistochemistry (2). Different anti-ErbB2 antibodies used for immunohistochemistry staining have also been shown to produce different degrees of ErbB2 staining in tumors, even in the presence of gene amplification (3), although applying calibration may help in minimizing those differences (4). The HercepTest (Dako) using Dako antibody was proposed as the standardized immunohistochemistry method to overcome the problem of interlaboratory variations. The scoring system uses the intensity of ErbB2 staining as its basis, and an ErbB2-positive tumor is defined as a tumor with greater than $10 \%$ of cells stained $3+(5)$. Despite use of the HercepTest, there still was a high discrepancy between local and central ErbB2 testing in the N9831 Intergroup Adjuvant Trial, with a concordance of only $81.6 \%$ for a diagnostic test for the presence of the ErbB2 protein (6). The American Society of Clinical Oncology and College of American Pathologists recommended an algorithm defining positive, equivocal, and negative values for both ErbB2 protein expression and gene amplification. A positive ErbB2 result from immunohistochemistry staining is defined by uniform, intense membrane staining of more than $30 \%$ of invasive tumor cells instead of the original $10 \%$ (5). However, despite this new algorithm and definition, not all laboratories have adopted this new guideline and there still are variable results in ErbB2 testing among laboratories. Furthermore, both the old and the new "ErbB2 counting" definitions have problems in detecting subtle ErbB2 changes induced by the treatment. For example, if trastuzumab decreases ErbB2 staining from $100 \%$ of the cells to $40 \%$, both the old and the new ErbB2 definition will score pre- and post-treatment samples as "positive" and may fail to detect ErbB2 changes after trastuzumab treatment.

After trastuzumab treatment, we found in human breast carcinoma BT474 xenografts a significant reduction of tracer uptake related to ErbB2 decrease rather than tumor size reduction (7). The observable reduction in PET signals could be due to partial-volume effect, but this possibility is rather unlikely since the images were acquired with high contrast and in the absence of background activity. When large enough regions are drawn around the tumor, the partial-volume effect does not cause any loss of signal, and the signal that is measured indicates the actual activity distribution. Moreover, for PET quantification, we deliberately chose the value related to maximum counts per pixel within the tumor that is least affected by partial-volume effect. Importantly, we have also shown that tumor ErbB2 membrane staining and PET changes correlated with tumor volume after 5 doses of trastuzumab treatment (7). There was a correlation between PET and immunohistochemistry, and the radionuclide concentrations measured with PET agreed with the radioactivity concentrations obtained by $\gamma$-counting (data were not presented). Although there was a large overlap in ErbB2 staining between the trastuzumab-treated and control groups, we found a significant reduction of ErbB2 downregulation after 5 doses of trastuzumab. This finding is consistent with several cell line experiments from different groups finding that trastuzumab downregulates ErbB2 receptors (8-10). After a single dose of trastuzumab, we could clearly see the differences in ErbB2 membrane staining between control and trastuzumab-treated samples, but the intensity percentage scoring failed to detect these changes (7).

The dose and duration of trastuzumab will clearly affect the amount of ErbB2 downregulation and the detection of ErbB2 changes by immunohistochemistry. In our paper, the dose of trastuzumab was deliberately high $(50 \mathrm{mg} / \mathrm{kg}$ loading dose followed by 4 more doses of $25 \mathrm{mg} / \mathrm{kg}$ each) to ensure that changes in receptor expression ErbB2 would be possible (7). Reddy et al. (11) treated BT474 xenografts with a lower dose of $10 \mathrm{mg} / \mathrm{kg}$ for only $6 \mathrm{~d}$. They found a decrease in PET tracer using C6.5 diabody but could not detect any ErbB2 changes by immunohistochemistry. They concluded that "The exact mechanism by which trastuzumab treatment inhibits $\mathrm{C} 6.5 \mathrm{db}$ binding is not yet understood." On the other hand, McLarty et al. (12) reported that trastuzumab reduced ErbB2 membrane staining in SKBR3 cells and in MDAMB361 and MDAMB361 xenograft models. In this case, mice were treated only with $4 \mathrm{mg}$ of trastuzumab per kilogram for $3 \mathrm{~d}$ or $3 \mathrm{wk}$. At $3 \mathrm{~d}$, the authors did not see ErbB2 membrane changes, but 3 wk later immunohistochemistry analysis of tumor tissues indicated significant ErbB2 downregulation, associated with almost complete eradication of viable tumor cells. This finding is consistent with our study as we did not see a difference in intensity percentage scoring after a single dose of trastuzumab (7); we observed differences in ErbB2 membrane staining after 5 doses of the drug (7). We believe that the differences seen in ErbB2 staining between Reddy et al. (11), McLarty et al. (12), and our study (7) may be related to the dose and duration of trastuzumab used. However, the differences may also be related to the ErbB2 testing methods and the scoring criteria, which could not detect subtle ErbB2 changes after trastuzumab treatment.

Trastuzumab was used as monotherapy before surgery in patients with primary operable ErbB2-positive breast tumors in a pilot study by Gennari et al. (13). They observed no change in ErbB2-positive staining using monoclonal antibody CB11 in the trastuzumab-treated samples. However, they provided figures from only 1 patient, as shown in their Figures 2A and 2B (13). Although these figures suggest some changes between pre- and posttreatment samples, the authors found no variations in the ErbB2 status (13). Furthermore, the use of a different anti-ErbB2 antibody and different scoring criteria may also have contributed 
to failure to detect ErbB2 changes between pre- and posttreatment samples.

Tagliabue et al. state in their letter that no changes in ErbB2 receptor status evaluated by immunohistochemistry were found in most patients with operable (14) or locally advanced (15) ErbB2-positive breast cancers after neoadjuvant exposure to trastuzumab in combination with chemotherapy. In the neoadjuvant study by Harris et al. (14), the ErbB2 status was based on the HercepTest, and 4 of 18 patients had lower immunohistochemistry scores. It is important to emphasize that HerceptTest criteria (i.e., $10 \%$ of cells positive) are not sensitive in detecting subtle ErbB2 changes induced by treatment. Therefore, it is possible that the HerceptTest could pick up ErbB2 changes in only a few patients because of the scoring criteria used. Mohsin et al. (15) used the Allred scoring system for ErbB2 changes between baseline and after 1 or $3 \mathrm{wk}$ of treatment and did not see a difference. However, this is not the standard method for ErbB2 testing and may not be able to differentiate between a weak ErbB2 intensity present in the whole tumor mass and a high ErbB2 intensity in just certain parts of the tumor, further underscoring the problems of using immunohistochemistry as the only screening test.

Mittendorf et al. (10) reported ErbB2 gene amplification loss in 1 of 3 patients, although the protein level measured by immunohistochemistry was not shown. It could be interesting to correlate gene level with protein expression. Tagliabue and colleagues argued that this loss of ErbB2 amplification is due to selection of ErbB2-negative cells rather than trastuzumab-induced ErbB2 downmodulation. Although we agree that selection of ErbB2-negative cells is a possibility, it is also possible that trastuzumab downregulates ErbB2 receptors resulting in tumor shrinkage but there is clonal expansion of the other ErbB2-negative cells.

We did not find a correlation between the impaired Affibody localization in xenografts and decreased vascularization. In fact, we saw the highest vessel count in those tumors with greater ErbB2 loss as assessed by PET although an elevated number of vessels was found only in the group of animals showing a dramatic decrease in ${ }^{18} \mathrm{~F}-\mathrm{FBEM}-\mathrm{HER}_{2: 342}$-Affibody uptake $(\mathrm{PET}[\% \mathrm{ID} / \mathrm{g}] \leq 0.55)$. We confirmed that the tumor size was not related to the average vessel count per field, and thus, we did not simply select tumors that responded to trastuzumab because of a better vascularization.

Regarding the comment by Tagliabue et al. citing their recent report that "tumor shrinkage induced by trastuzumab-containing therapy can sometimes be followed by an inflammatory reaction that masks any decrease in neoplastic mass" (16), that report was a retrospective study assessing the use of trastuzumab beyond progression, and therapy included a variety of additional chemotherapy agents. ErbB2 levels were not evaluated in progressing tumors.

In summary, we agree that there have been various controversial reports on the effect of trastuzumab on ErbB2 receptor downregulation in cell lines, xenograft models, and human studies. Modeling the therapeutic activity of any antibody in human xenografts is challenging. Their vascular system is derived from the host. Subcutaneous transplants do not recapitulate the systemic and metabolic effects of spontaneous cancers, and they fail to capture the contribution of the immune system, which is saved in syngeneic systems. Therefore, these models may not completely mimic the response in human patients.

The particular animal model, the dose of trastuzumab, and the duration of trastuzumab could all affect the amount of ErbB2 downregulation by trastuzumab observed in preclinical studies. Furthermore, differences in immunohistochemistry staining techniques, antibodies used, and scoring criteria could account for differing results in the assessment of ErbB2 status by immunohistochemistry in both animal and human studies.

\section{REFERENCES}

1. Perez EA, Suman VJ, Davidson NE, et al. HER2 testing by local, central, and reference laboratories in specimens from the North Central Cancer Treatment Group N9831 intergroup adjuvant trial. J Clin Oncol. 2006;24:3032-3038.

2. Yaziji H, Goldstein LC, Barry TS, et al. HER-2 testing in breast cancer using parallel tissue-based methods. JAMA. 2004;291:1972-1977.

3. Mitchell MS, Press MF. The role of immunohistochemistry and fluorescence in situ hybridization for HER2/neu in assessing the prognosis of breast cancer. Semin Oncol. 1999;26(4, suppl 12)108-116.

4. Vincent-Salomon A, MacGrogan G, Couturier J, et al. Calibration of immunohistochemistry for assessment of HER2 in breast cancer: results of the French multicentre GEFPICS study. Histopathology. 2003;42:337-347.

5. Wolff AC, Hammond ME, Schwartz JN, et al. American Society of Clinical Oncology/College of American Pathologists guideline recommendations for human epidermal growth factor receptor 2 testing in breast cancer. J Clin Oncol. 2007;25:118-145.

6. Perez EA, Suman VJ, Davidson NE, et al. HER2 testing by local, central, and reference laboratories in specimens from the North Central Cancer Treatment Group N9831 Intergroup Adjuvant Trial. J Clin Oncol. 2006;24:3032-3038.

7. Kramer-Marek G, Gijsen M, Kiesewetter DO, et al. Potential of PET to predict the response to trastuzumab treatment in an ErbB2-positive human xenograft tumor model. J Nucl Med. 2012;53:629-637.

8. Gijsen M, King P, Perera T, et al. HER2 phosphorylation is maintained by a PKB negative feedback loop in response to anti-HER2 Herceptin in breast cancer. PLoS Biol. 2010;8:e1000563.

9. Cuello M, Ettenberg SA, Clark AS, et al. Down-regulation of the erbB-2 receptor by trastuzumab (Herceptin) enhances tumor necrosis factor-related apoptosisinducing ligand-mediated apoptosis in breast and ovarian cancer cell lines that overexpress erbB-2. Cancer Res. 2001;61:4892-4900.

10. Mittendorf EA, Wu Y, Scaltriti M, et al. Loss of HER2 amplification following trastuzumab-based neoadjuvant systemic therapy and survival outcomes. Clin Cancer Res. 2009;15:7381-7388.

11. Reddy S, Shaller CC, Doss M, et al. Evaluation of the anti-HER2 C6.5 diabody as a PET radiotracer to monitor HER2 status and predict response to trastuzumab treatment. Clin Cancer Res. 2011;17:1509-1520.

12. McLarty K, Cornelissen B, Cai Z, et al. Micro-SPECT/CT with ${ }^{111}$ In-DTPApertuzumab sensitively detects trastuzumab-mediated HER2 downregulation and tumor response in athymic mice bearing MDA-MB-361 human breast cancer xenografts. J Nucl Med. 2009;50:1340-1348.

13. Gennari R, Ménard S, Fagnoni F, et al. Pilot study of the mechanism of action of preoperative trastuzumab in patients with primary operable breast tumors overexpressing HER2. Clin Cancer Res. 2004;10:5650-5655.

14. Harris LN, You F, Schnitt SJ, et al. Predictors of resistance to preoperative trastuzumab and vinorelbine for HER2-positive early breast cancer. Clin Cancer Res. 2007;13:1198-1207.

15. Mohsin SK, Weiss HL, Gutierrez MC, et al. Neoadjuvant trastuzumab induces apoptosis in primary breast cancers. J Clin Oncol. 2005;23:24602468 .

16. Campiglio M, Bufalino R, Sandri M, et al. Increased overall survival independent of RECIST response in metastatic breast cancer patients continuing trastuzumab treatment: evidence from a retrospective study. Breast Cancer Res Treat. 2011;128: $147-154$.

Jacek Capala*

Anthony Kong

Gabriela Kramer-Marek

Merel Gijsen

National Institutes of Health

10 Center Dr., Building 10, Room B3B69D

Bethesda, MD 20892

E-mail: capalaj@mail.nih.gov

Published online Sep. 4, 2012

DOI: 10.2967/jnumed.112.109496 
The Value of ${ }^{18}$ F-FDG PET/CT in the Assessment of Cardiac Malignancy Remains to Be Defined

TO THE EDITOR: We read with great interest the recent article by Rahbar et al. titled "Differentiation of Malignant and Benign Cardiac Tumors Using ${ }^{18} \mathrm{~F}$-FDG PET/CT" (1). The paper is interesting because diagnosis of cardiac malignancy is difficult and poorly defined. For example, it has been estimated that in most melanoma patients with cardiac metastases, the metastases remain undiagnosed (2). However, several concerns in this paper need to be discussed and clarified.

The first is that special patient preparation is required for detecting cardiac malignancy. It is well known that ${ }^{18} \mathrm{~F}$-FDG uptake in the heart is highly heterogeneous. Fasting for $6 \mathrm{~h}$, as used in the study of Rahbar et al., is not enough to significantly suppress physiologic ${ }^{18} \mathrm{~F}$-FDG uptake of the heart and thus does not offer the ability to differentiate malignancy from physiologic activity (3). We personally examined the ${ }^{18} \mathrm{~F}-\mathrm{FDG}$ PET/CT images of 27 patients who had fasted overnight (10-14 h), and we found that ${ }^{18} \mathrm{~F}$-FDG uptake in the myocardium (the lateral wall of the left ventricle) varied significantly, with maximum standardized uptake value (SUV) ranging from 2.1 to 27.15 (mean $\pm \mathrm{SD}, 11.22 \pm 7.71$; with $13 / 27$ having an SUV $>10$ and only $8 / 27$ having an SUV $<5$ ), consistent with reports in the literature $(3,4)$. It is likely that the difference between benign and malignant cardiac tumors is less than the variation in myocardial ${ }^{18} \mathrm{~F}$-FDG uptake in healthy persons. To solve this problem, a low-carbohydrate, high-fat, high-protein diet has been proposed in addition to overnight fasting to minimize background ${ }^{18} \mathrm{~F}$-FDG uptake in the myocardium (2,5-7). This diet significantly reduces but still does not allow complete suppression of myocardial ${ }^{18} \mathrm{~F}-$ FDG uptake.

The authors performed a receiver-operating-characteristic analysis and obtained cutoff maximum SUVs of 3.5 (with a sensitivity of $100 \%$ and specificity of $86 \%$ ) and 4.6 (with a sensitivity of $94 \%$ and specificity of $100 \%$ ) with high diagnostic accuracy. The authors did not specify for what category the sensitivity and specificity were, and we assume that these sensitivity and specificity values were for identifying malignant cases from a total of benign and malignant cardiac tumor cases. However, these seemingly excellent results are misleading and have limited clinical value. The receiver-operatingcharacteristic analysis was performed on patients with known cardiac tumors. As such, the sensitivity and specificity obtained in this paper are applicable only to a patient population with known cardiac tumors and cannot be applied to a general patient population or even to patients with suspected cardiac malignancy. Because the prevalence of cardiac malignancy is low in the general patient population, these cutoff SUVs as described in this article would lead to high false-positive results, although use of these criteria in patients highly suspected of having cardiac malignancy is possible and worth further investigation. Even in patients prepared with a low-carbohydrate, high-fat, high-protein diet and overnight fasting, variation in ${ }^{18} \mathrm{~F}-\mathrm{FDG}$ uptake in the heart remains high. For example, Williams et al. (5) reported a cardiac maximum SUV of $3.9 \pm 3.6$ (average $\pm \mathrm{SD}$ ) in 60 patients, with 16 patients (26.7\%) having a maximum SUV above 4 and 3 patients (5\%) having a maximum SUV above 15 . The heterogeneity of cardiac ${ }^{18} \mathrm{~F}-\mathrm{FDG}$ uptake and the low prevalence of cardiac tumors make the accurate detection of cardiac tumors (either benign or malignant) on ${ }^{18} \mathrm{~F}$-FDG PET problematic. More useful would be a receiver-operating-characteristic analysis performed on a patient population representative of clinical practice.

Other causes of increased cardiac ${ }^{18} \mathrm{~F}-\mathrm{FDG}$ uptake should also be considered. For example, sarcoidosis lesions often have increased ${ }^{18}$ F-FDG uptake comparable to that of malignancy. With an estimated prevalence of cardiac involvement of at least $25 \%$ (8), cardiac sarcoidosis is probably a more common cause of increased uptake in the heart, further complicating the interpretation of an ${ }^{18}$ F-FDG PET study of the heart. Correlation with the patient's history and other imaging findings will be critical for accurate diagnosis on ${ }^{18} \mathrm{~F}$-FDG PET.

Finally, the authors did not clarify whether biopsy of heart lesions was performed on all patients and whether biopsy was performed before or after ${ }^{18}$ F-FDG PET. The authors stated that the grouping of patients was based on "the histologic characterization of the surgically resected cardiac tumors or tumor biopsies." Apparently, then, the pathologic findings were available for this analysis, which may lead to significant bias in this study.

\section{REFERENCES}

1. Rahbar K, Seifarth H, Schäfers M, et al. Differentiation of malignant and benign cardiac tumors using ${ }^{18}$ F-FDG PET/CT. J Nucl Med. 2012;53:856-863.

2. Cheng G, Newberg A, Alavi A. Metastatic melanoma causing complete atrioventricular block - the role of FDG PET/CT in diagnosis. Clin Imaging. 2011;35:312-314.

3. Kaneta T, Hakamatsuka T, Takanami K, et al. Evaluation of the relationship between physiological FDG uptake in the heart and age, blood glucose level, fasting period, and hospitalization. Ann Nucl Med. 2006;20:203-208.

4. Inglese E, Leva L, Matheoud R, et al. Spatial and temporal heterogeneity of regional myocardial uptake in patients without heart disease under fasting conditions on repeated whole-body ${ }^{18}$ F-FDG PET/CT. J Nucl Med. 2007;48:1662-1669.

5. Williams G, Kolodny GM. Suppression of myocardial ${ }^{18} \mathrm{~F}-\mathrm{FDG}$ uptake by preparing patients with a high-fat, low-carbohydrate diet. AJR. 2008;190:W151-W156.

6. Wykrzykowska J, Lehman S, Williams G, et al. Imaging of inflamed and vulnerable plaque in coronary arteries with ${ }^{18} \mathrm{~F}-\mathrm{FDG}$ PET/CT in patients with suppression of myocardial uptake using a low-carbohydrate, high-fat preparation. J Nucl Med. 2009;50:563-568.

7. Harisankar CNB, Mittal BR, Agrawal KL, Abrar ML, Bhattacharya A. Utility of high fat and low carbohydrate diet in suppressing myocardial FDG uptake. $J$ Nucl Cardiol. 2011;18:926-936.

8. Youssef G, Leung E, Mylonas I, et al. The use of ${ }^{18} \mathrm{~F}-\mathrm{FDG}$ PET in the diagnosis of cardiac sarcoidosis: a systematic review and metaanalysis including the Ontario experience. J Nucl Med. 2012;53:241-248.

\section{Gang Cheng* \\ Abass Alavi \\ *Philadelphia VA Medical Center \\ 3900 Woodland Ave. \\ Philadelphia, PA 19104 \\ E-mail: gangcheng99@yahoo.com}

Published online Aug. 22, 2012.

DOI: 10.2967/jnumed.112.109611

REPLY: We thank Drs. Cheng and Alavi for adding and corroborating interesting points of discussion. We completely agree with the authors that the sensitivity and specificity of ${ }^{18}$ F-FDG PET would be much lower in patients without a prior diagnosis of a cardiac tumor by morphologic imaging. Our results are restricted to patients with known cardiac tumors. ${ }^{18} \mathrm{~F}$-FDG PET is certainly not going to be the first-line procedure for excluding cardiac involvement in patients with known or suspected malignancy elsewhere.

Physiologic myocardial uptake was not so great an obstacle as suggested in the letter. It has to be kept in mind that the location 
of the tumor was known by morphologic imaging. In some cases, regional physiologic uptake in the myocardium was observed, but as reported, the vicinity of the tumors showed a mean myocardial uptake of as low as $2.1 \pm 0.6$ standardized uptake value (SUV) (1). Peritumoral myocardial dysfunction might be discussed as an explanation of this finding, but in the absence of further evidence this assumption was not discussed in the article.

Nevertheless, we support the concept of a prolonged fasting period.

Sarcoidosis is certainly a condition that may mimic malignant disease. Patient inclusion criteria were primarily based on morphologic imaging. The probability of sarcoidosis was low according to imaging and clinical information. The differential diagnosis was therefore no major problem in this series of patients. In that context it has to be emphasized that sufficient results in functional imaging can be obtained only with state-of-the-art morphologic imaging techniques in the background.

Tumor biopsy was performed before ${ }^{18} \mathrm{~F}-\mathrm{FDG}$ PET/CT in 3 of 24 patients: almost 2 mo before PET/CT in one of these patients and within $1 \mathrm{wk}$ in the other two. In all patients, the tumors had a malignant histology, and the smallest tumor had a maximum diameter of $5.6 \mathrm{~cm}$. There is no evidence that inclusion of these 3 patients systematically affect the results of the study.
We completely agree with Drs. Cheng and Alavi that the proposed cutoff of 3.5 SUV cannot be applied to an unselected population to screen for myocardial malignancy. Maximum SUV depends on many factors such as scanner resolution, lesion size, scan delay after injection, and the use of motion correction. The cutoff is valid only in the technical and clinical setting described in detail in the article. We thank Drs. Cheng and Alavi for emphasizing this important issue.

\section{REFERENCE}

1. Rahbar K, Seifarth H, Schäfers M, et al. Differentiation of malignant and benign cardiac tumors using ${ }^{18}$ F-FDG PET/CT. J Nucl Med. 2012;53:856-863.

\author{
Kambiz Rahbar* \\ Otmar Schober \\ Matthias Weckesser \\ *University Hospital Münster \\ Albert-Schweitzer-Campus 1 \\ 48149 Münster, Germany \\ E-mail: rahbar@uni-muenster.de
}

Published online Aug. 23, 2012.

DOI: 10.2967/jnumed.112.110676

\section{Erratum}

The authors of "Impact of Dynamic ${ }^{18}$ F-FDG PET on the Early Prediction of Therapy Outcome in Patients with High-Rish Soft-Tissue Sarcomas After Neoadjuvant Chemotherapy: A Feasibility Study" (Dimitrakopoulou-Strauss et al. J Nucl Med. 2010;51:551-558) regret that Table 2 contained some errors. The corrected table appears below.

TABLE 2

Results of Linear Discriminant Analysis with Equal Prior Probabilities Based on ${ }^{18} \mathrm{~F}-\mathrm{FDG}$ Parameters of First PET Study (1) or Second PET Study (2) or Combination of Both Studies

\begin{tabular}{lcrrrr}
\hline \multicolumn{1}{c}{ Parameter } & PPV & \multicolumn{1}{c}{ NPV } & \multicolumn{1}{c}{ Sensitivity } & \multicolumn{1}{c}{ Specificity } & Accuracy \\
\hline 1: SUV & $9 / 15(60.00 \%)$ & $7 / 10(70.00 \%)$ & $9 / 12(75.00 \%)$ & $7 / 13(54.00 \%)$ & $16 / 25(64.00 \%)$ \\
1: SUV, VB, k1, k3, FD & $9 / 11(81.81 \%)$ & $11 / 14(78.57 \%)$ & $9 / 12(75.00 \%)$ & $11 / 13(84.62 \%)$ & $20 / 25(80.00 \%)$ \\
2: SUV & $10 / 16(62.5 \%)$ & $6 / 8(75.00 \%)$ & $10 / 12(83.33 \%)$ & $6 / 12(50.00 \%)$ & $16 / 24(66.70 \%)$ \\
2: SUV, influx & $8 / 10(80.00 \%)$ & $10 / 14(71.43 \%)$ & $8 / 12(67.00 \%)$ & $10 / 12(83.30 \%)$ & $18 / 24(75.00 \%)$ \\
2: FD, k4 & $9 / 11(81.81 \%)$ & $10 / 13(76.92 \%)$ & $9 / 12(75.00 \%)$ & $10 / 12(83.30 \%)$ & $19 / 24(79.20 \%)$ \\
1 + 2: SUV & $9 / 14(64.30 \%)$ & $7 / 10(70.00 \%)$ & $9 / 12(75.00 \%)$ & $7 / 12(58.33 \%)$ & $16 / 24(66.70 \%)$ \\
1 + 2: SUV, influx & $11 / 14(78.60 \%)$ & $9 / 10(90.00 \%)$ & $11 / 12(91.67 \%)$ & $9 / 12(75.00 \%)$ & $20 / 24(83.33 \%)$ \\
\% change SUVmax & $8 / 14(57.14 \%)$ & $6 / 10(60.00 \%)$ & $8 / 12(66.67 \%)$ & $6 / 12(50.00 \%)$ & $14 / 24(58.33 \%)$
\end{tabular}

Groups were defined according to histologic classification of $10 \%$ variable tumor tissue. $\mathrm{PPV}=$ positive predictive value; NPV = negative predictive value. 